\title{
CLOSED DIVISION OF THE FLEXOR TENDON SHEATH FOR TRIGGER FINGER
}

\author{
SHAW-RUEY LYU
}

From Chiayi Christian Hospital, Taiwan

\begin{abstract}
Closed tenotomy was used to treat triggering of the fingers and thumb in 54 patients. In 56 digits the method was successful; in seven it was a simple matter to proceed to open tenotomy. With experience, the closed procedure can be completed within minutes without risk of damaging the digital nerves.
\end{abstract}

Stenosing tenosynovitis of the thumb or finger flexors is a common cause of pain and disability (Lipscomb 1959; Medl 1970; Weilby 1970). Surgical division of the Al pulley is a well-known cure but the conventional open method can cause discomfort and is not free from complications (Miller 1950; Lapidus 1953; Fahey and Bollinger 1954). A tourniquet is sometimes needed to control bleeding and ensure the safety of the procedure.

Special instruments and a new technique have been developed to divide the pulley percutaneously and thereby avoid these problems.

\section{MATERIALS AND METHODS}

Patients. From April 1988 to November 1990, 63 trigger fingers or thumbs in 54 consecutive patients were treated by this method. There were 33 women and 21 men; their ages ranged from 38 to 68 years (mean 53). There were 16 thumbs, 12 index fingers, 11 long fingers, 21 ring fingers and three little fingers. All the patients had experienced typical symptoms of painful triggering or snapping when the involved digit was flexed and extended and all had local tenderness or a palpable nodule in the palm. The trigger phenomenon could not be elicited at the time of the operation in seven patients.

The indications for surgical treatment included persistence of symptoms for more than three months, involvement of several digits, recurrence after local injection or splinting, and/or willingness to accept the procedure as primary treatment when its simplicity and effectiveness had been explained.

S-R. Lyu, MD, Head of Department

Department of Orthopaedics, Chiayi Christian Hospital, 539 Chung Hsiao Road, Chiayi, Taiwan, Republic of China.

(C) 1992 British Editorial Society of Bone and Joint Surgery $0301-620 X / 92 / 3352 \$ 2.00$

J Bone Joint Surg [Br] 1992; 74-B : 418-20
Instruments. The instruments used were a pulley hook and a curved-blade knife (Fig. 1). The pulley hook is a small aneurysm needle appropriately bent. Its width is about $2 \mathrm{~mm}$ and it has a groove along its concave surface.

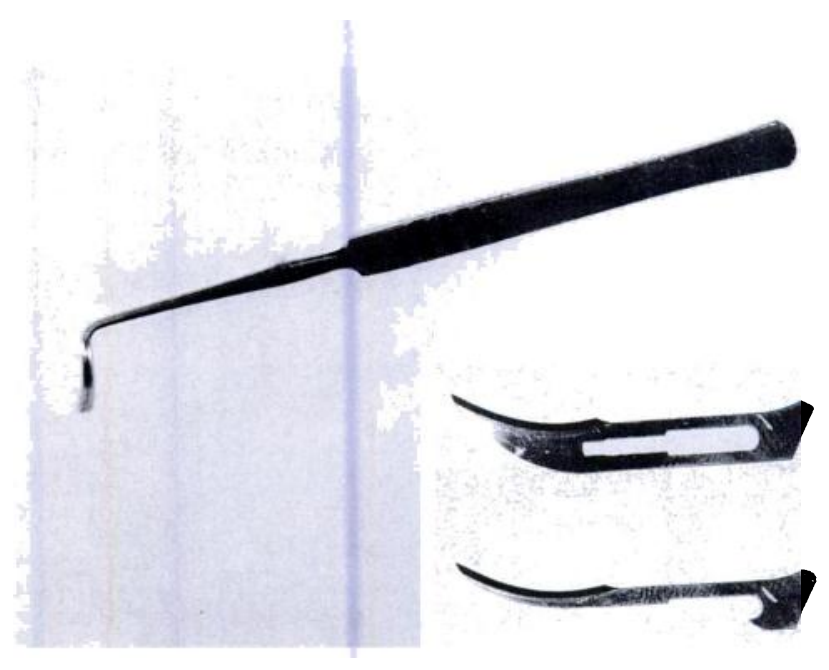

Fig. 1

The modified aneurysm needle and the curved blade used for closed tenotomy of the Al pulley.

The curved blade can be easily fashioned with an electric grindstone from a number- 12 surgical blade.

Operative technique. Precise location of the skin incision is the most important determinant of success. It should be placed at the proximal edge of the first annular pulley of the flexor tendon. This almost exactly coincides with the distal palmar crease in the fourth and fifth rays, and the proximal palmar crease in the index finger, and lies halfway between the two creases in the middle finger. The proximal edge of the annulus of the flexor pollicis longus is directly under the metacarpophalangeal flexion crease of the thumb (Fig. 2, Lorthioir 1958). Having carefully selected the site of the incision, a 25-gauge 
needle is introduced and about $0.5 \mathrm{ml}$ of $1 \%$ lignocaine (lidocaine) is infiltrated into the skin and the tissues around the tendon sheath. A 2 to $3 \mathrm{~mm}$ transverse incision is made with a number-11 scalpel blade over the needle hole and the tip of the pulley hook is pushed into the small wound until it touches the anterior surface of the tendon sheath. The proximal edge of the first annular pulley is detected by sliding the tip of the hook backward and forward on the tendon sheath and the hook is then thrust with some force between the pulley and the flexor tendon (Fig. 3). The curved-blade knife is introduced along the groove on the concave side of the hook to divide the Al pulley. The patient is asked to flex and extend the finger to verify the success of the procedure. No

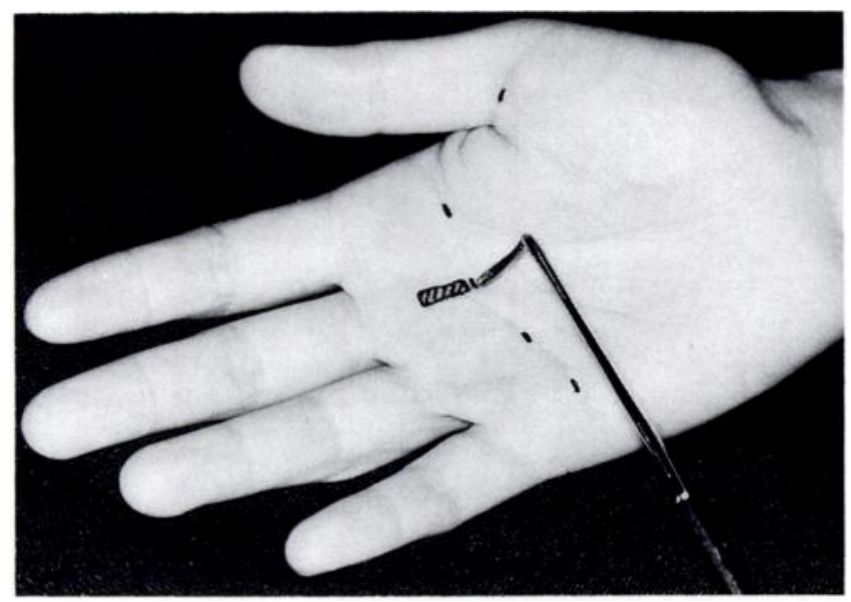

Fig. 2

Surface anatomy of the location of the proximal edge of the Al pulley.

tourniquet is used and the small wound does not need to be sutured.

If a surgeon, inexperienced in the technique, fails to introduce the pulley hook successfully by the percutaneous method, he can enlarge the incision and complete the operation under direct vision.

A small dressing is used to cover the wound for two days and the patient then returns to normal activities.

\section{RESULTS}

In our series of 63 operations an open technique had to be performed on seven occasions. Two occurred at the beginning of the series and were due to inexperience. On the other five occasions the trigger phenomenon could not be demonstrated at the time of operation and the surgeon could not therefore verify the success of the closed method. Direct inspection showed that four of the five pulleys had in fact been satisfactorily divided.

All the patients were reviewed once a week for at least four weeks postoperatively. Most resumed their work immediately after the procedure and had no more than mild pain for a few days. In three patients pain and local swelling persisted for up to three weeks.

A telephone interview with $\mathbf{4 8}$ of the 54 patients, at an average of 11 months after operation (six to 24), showed that all were satisfied with the result. The treated digits were pain free and no longer clicked or locked. One woman complained of mild discomfort over a hypertrophic scar in her palm.

\section{DISCUSSION}

Lorthioir (1958) was the first to propose a percutaneous technique. He used a fine tenotome to pierce the skin and

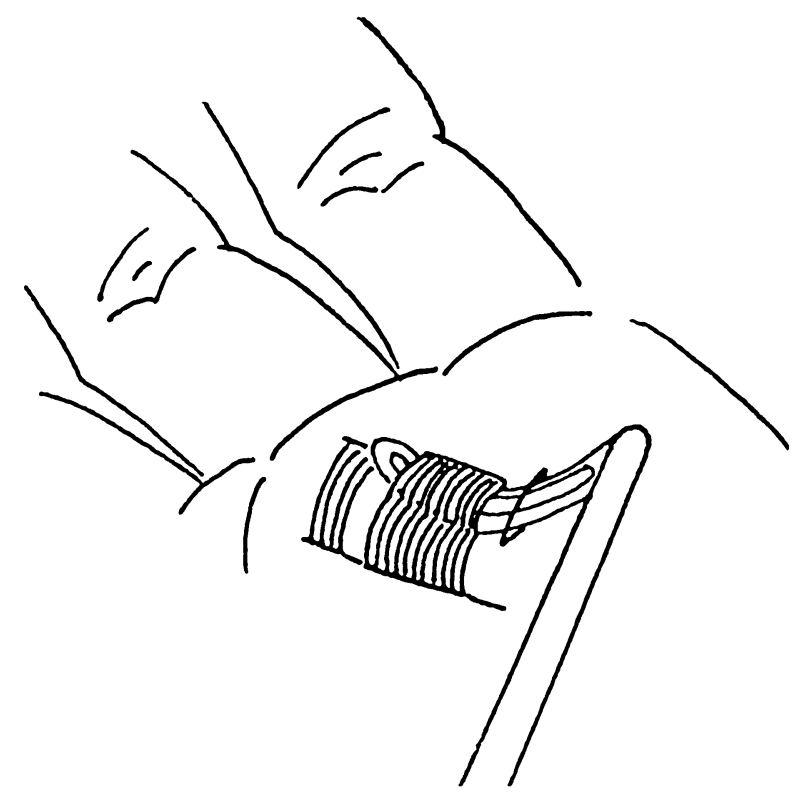

Fig. 3

The modified aneurysm needle in place deep to the Al pulley.

cut the Al pulley, but although he obtained good results in 52 patients, his technique was not widely adopted, perhaps because the 'blind' method seemed too dangerous.

Our closed method was tested in several cadavers before clinical application and dissection was performed after each procedure to verify the reproducibility of the operation. Division of the Al pulley was never associated with simultaneous division of the A2 pulley. The digital nerves were also never in danger; they course along the sides of the flexor tendon and should not be injured through the tiny wound which does not exceed the width of the tendon. In the thumb, the most vulnerable structure is the radial digital nerve which passes diagonally across the flexor hallucis longus tendon from the ulnar to the radial side. The site of this crossing is a few millimetres 
proximal to the metacarpophalangeal flexion crease of the thumb. Since the puncture wound is made in the flexion crease the nerve should not be at risk. Once the hook is inserted under the pulley the nerve lies proximally and is further protected as the curved blade is pushed along the groove in the hook.

The operation was more easily performed on patients than on cadavers because the pathologically thickened Al pulley is more easily located. The surgeon should, however, first practise on cadavers and then use the technique in an operating theatre, in case it is necessary to proceed to an open division.

No benefits in any form have been received or will be received from a commercial party related directly or indirectly to the subject of this article.

\section{REFERENCES}

Fahey JJ, Bollinger JA. Trigger-finger in adults and children. $J$ Bone Joint Surg [Am] 1954; 36-A :1200-18.

Lapidus PW. Stenosing tenovaginitis. Surg Clin North [Am] 1953; 33:1317-47.

Lipscomb PR. Tenosynovitis of the hand and the wrist: carpal tunnel syndrome, deQuervain's disease, trigger digit. Clin Orthop 1959; 13:164-80.

Lorthioir J. Surgical treatment of trigger-finger by a subcutaneous method. J Bone Joint Surg [ Am] 1958; 40-A :793-5.

Medl WT. Tendonitis, tenosynovitis, 'trigger finger', and Quervain's disease. Orthop Clin North Am 1970; $1: 375-82$.

Miller LF. Stenosing tendovaginitis: a survey of findings and treatment in 49 cases. Indust Med 1950; 19:465-7.

Weilby $A$. Trigger finger: incidence in children and adults and the possibility of a predisposition in certain age groups. Acta Orthop Scand $1970 ; 41: 419-27$. 